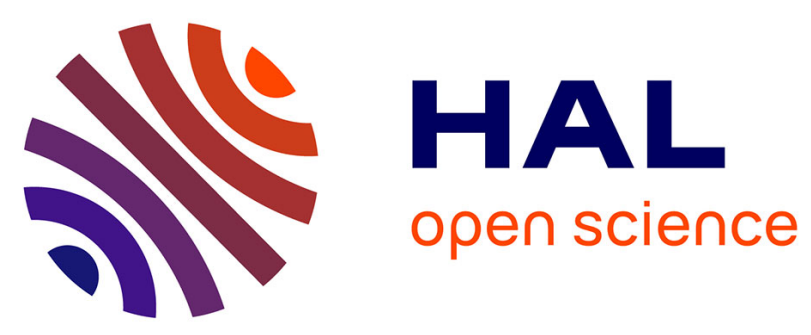

\title{
Efficient Resource Allocation for Multi-tenant Monitoring of Edge Infrastructures
}

Mohamed Abderrahim, Meryem Ouzzif, Karine Guillouard, Jérôme François, Adrien Lebre, Charles Prud'Homme, Xavier Lorca

\section{To cite this version:}

Mohamed Abderrahim, Meryem Ouzzif, Karine Guillouard, Jérôme François, Adrien Lebre, et al.. Efficient Resource Allocation for Multi-tenant Monitoring of Edge Infrastructures. PDP 2019 - 27th Euromicro International Conference on Parallel, Distributed and Network-Based Processing, Feb 2019, Pavie, Italy. pp.1-8, 10.1109/EMPDP.2019.8671621 . hal-01987946

\section{HAL Id: hal-01987946 \\ https://hal.inria.fr/hal-01987946}

Submitted on 21 Jan 2019

HAL is a multi-disciplinary open access archive for the deposit and dissemination of scientific research documents, whether they are published or not. The documents may come from teaching and research institutions in France or abroad, or from public or private research centers.
L'archive ouverte pluridisciplinaire HAL, est destinée au dépôt et à la diffusion de documents scientifiques de niveau recherche, publiés ou non, émanant des établissements d'enseignement et de recherche français ou étrangers, des laboratoires publics ou privés. 


\section{Efficient Resource Allocation for Multi-tenant Monitoring of Edge Infrastructures}

\author{
Mohamed Abderrahim, \\ Meryem Ouzzif and Karine Guillouard \\ Orange Labs, France \\ firstname.lastname@orange.com
}

\author{
Jérôme François \\ Inria, France \\ firstname.lastname@inria.fr
}

Xavier Lorca

IMT Mines Albi, France

firstname.lastname@mines-albi.fr

\author{
Adrien Lebre \\ and Charles Prud'homme \\ IMT Atlantique, France \\ firstname.lastname@imt-atlantique.fr
}

\begin{abstract}
By relying on small sized and massively distributed infrastructures, the Edge computing paradigm aims at supporting the low latency and high bandwidth requirements of the next generation services that will leverage IoT devices (e.g., video cameras, sensors). To favor the advent of this paradigm, management services, similar to the ones that made the success of Cloud computing platforms, should be proposed. However, they should be designed in order to cope with the limited capabilities of the resources that are located at the edge. In that sense, they should mitigate as much as possible their footprint. Among the different management services that need to be revisited, we investigate in this paper the monitoring one. Monitoring functions tend to become compute-, storage- and network-intensive, in particular because they will be used by a large part of applications that rely on real-time data. To reduce as much as possible the footprint of the whole monitoring service, we propose to mutualize identical processing functions among different tenants while ensuring their quality-of-service (QoS) expectations. We formalize our approach as a constraint satisfaction problem and show through micro-benchmarks its relevance to mitigate compute and network footprints.
\end{abstract}

\section{Introduction}

The proliferation of Internet of Things (IoT) applications [1], as well as the advent of new technologies such as Mobile Edge computing [2], Software-Defined Networking [3] and Network Function Virtualization [4] (NFV) have been accelerating the need for Edge computing infrastructures [5]. Meanwhile, progress on how to operate and use such infrastructures is marginal. Existing operational solutions such as Akamai+Cloudlet 1 or Amazon Lambda@Edge ${ }^{2}$ allow to run only domain-specific applications on infrastructures composed of centralized clouds and NFV-enabled hardware at the edge.

In order to satisfy the expectations of operators and users of Edge infrastructures, we claim that resource management services with similar capabilities that made the success

1. http://cloudlets.akamai.com

2. https://aws.amazon.com/lambda/edge/ of Cloud computing should be designed as follows. First, they should let an operator aggregate, supervise and expose the massively distributed resources of an Edge infrastructure. Secondly, they should let third party users implement new kinds of services on top of them. Reusing available management systems such as OpenStack or OpenMANO cannot be done in a straightforward manner as most of them have been designed for centralized data-centers [6]. In other words, they do not take into account network specifics (e.g., latency, bandwidth, intermittent connectivity) to handle the distribution of the Edge resources. Moreover, they do not consider mitigating the management footprint to cope with the limited capabilities of Edge resources.

Among the management services that should be revised, the monitoring service is an important one. It is mandatory for all the infrastructure tenants to detect faults, ensure security, observe the QoS and provision resources. Besides, it has to cope with the multitude of tenants resources that may be owned by the infrastructure operator (e.g., servers, routers, network links), by edge resources providers (e.g., home gateways, smartphones, laptops), by services providers (e.g., media contents, Web sites) and by services users (e.g., virtual machines, virtual network functions, temperature sensors). The large number of resources being observed leads to a large number of measurements to be processed and transmitted through the Edge infrastructure. For instance, the sensors observing Twitter's infrastructure generate 2.8 Billion measurements per minute [7] and a single sensor which monitors electricity consumption generates 50 Billion measurements per year [8].

In order to mitigate as much as possible the monitoring footprint on the Edge infrastructure, we aim at designing a monitoring as a service solution [9] to be offered to the infrastructure tenants according to the utility computing model. In addition to performing the processing required by the different tenants, it limits the processing redundancy. For instance, monitoring virtual servers may be of interest to both the infrastructure operator and a IaaS service user. In addition, the observations of an Internet-connected camera installed in a city may be of interest to both a police and a transportation office. Presently, such resources are monitored separately. As a result, a redundant processing is performed 
for each tenant. We aim at limiting such redundancies by designing a monitoring service that mutualizes processing among different tenants while keeping their functional and QoS requirements fully satisfied.

We proceed using elementary functions deduced from our previous work [10] to express the monitoring processing requests in a unified way. The best composition of functions is then achieved by mutualizing as many functions as possible among the different tenants while taking into account their QoS constraints and the infrastructure capabilities.

The contributions are: (i) the proposal of the monitoring functions mutualization among different tenants as an approach to reduce the monitoring footprint, (ii) the formalization of our approach as a constraint satisfaction problem that considers the Edge infrastructure capabilities and the tenants requirements, and (iii) the validation of our approach through different scenarios.

The rest of the paper is organized as follows. Section 2 details how we model the problem. Section 3 evaluates our approach. Section 4 discusses the related work. Finally, Section 5 concludes the paper and gives some perspectives for this work.

\section{The Mutualized Placement Problem}

Our approach of mutualizing identical processing and flows among different tenants is motivated by the multitenant aspect of the Edge infrastructure. In fact, the tenants sharing the same resources are likely to have overlapping monitoring requirements. By mutualising processing and flows among them, we aim to reduce the compute and the network footprint of the monitoring service. However, our approach is challenged by keeping each tenant requirement satisfied. For this purpose, two types of requirements should be considered. The first one is the functional requirement: the mutualization should not change the resulting processing that is exposed to each tenant. The second type is the QoS requirement: the mutualization should keep the end-to-end performance requirements (e.g., latency) satisfied for each tenant.

The users functional requirements consist of the preliminary measurements processing (e.g., aggregation, filtering, events triggering ...) that we identified in our previous study [10]. They may be expressed as chains of elementary algebraic functions such as aggregate, filter, join, split ... Thus, we model the functional requirements as a directed graph whose vertices represent the elementary functions and whose arcs represent the flow dependencies between them. To guarantee their satisfaction, we model the mutualization as an edge contraction [11] that deals with merging graph vertices according to specific constraints. In order to satisfy the QoS requirements, the infrastructure capabilities should be considered also. Thus, this comes down to a placement problem. We model it as an inexact graph matching [12] that consists in determining a mapping between two graphs having a different number of vertices. In our case, the first graph represents the merged users requirements graph and the second one represents the infrastructure. Both edge contraction and inexact graph matching have been proved to be NP-complete problems [13] [11].

Since the determination of the mutualized placement is constraint-oriented and NP-complete, we opt here for formalizing it as a constraint satisfaction problem [14]. Table 1 summarizes our model. In the following, it is detailed.

\begin{tabular}{|c|c|}
\hline \multicolumn{2}{|r|}{ Inputs } \\
\hline$P$ & The set of probe functions \\
\hline$M$ & The set of processing functions \\
\hline$U$ & The set of user functions \\
\hline$F$ & The set of all the functions: $F=P \cup M \cup U$ \\
\hline$R$ & The set of flows exchanged by the functions \\
\hline$l_{f f}$ & $\begin{array}{l}\text { For a given pair of } F \times F \text {, this function returns true } \\
\text { if both elements of the pair are identical (i.e., have the } \\
\text { same type and parameters). Otherwise, it returns false. }\end{array}$ \\
\hline$l_{f C}$ & $\begin{array}{l}\text { For a given element of } F \text {, this function returns the } \\
\text { required server capability by that element. }\end{array}$ \\
\hline$l_{f S}$ & $\begin{array}{l}\text { For a given element of } F \text {, this function returns the } \\
\text { server of } S \text { that should host it or null if there is no } \\
\text { constraint on the hosting server. }\end{array}$ \\
\hline$l_{f L}$ & $\begin{array}{l}\text { For a given element of } F \text {, this function returns the } \\
\text { tolerated latency between that element and the probes. }\end{array}$ \\
\hline$l_{o C}$ & $\begin{array}{l}\text { For a given flow of } F \times F \text {, this function returns the } \\
\text { required link capability by that flow. }\end{array}$ \\
\hline$G_{U}$ & $\begin{array}{l}\text { The users requirements modeling graph. } G_{U}=(F, R \text {, } \\
\left.l_{f C}, l_{f f}, l_{f S}, l_{f L}, l_{o C}\right)\end{array}$ \\
\hline$S$ & The set of servers \\
\hline$L$ & The set of network links \\
\hline$l_{s C}$ & $\begin{array}{l}\text { For a given server of } S \text {, this function returns the } \\
\text { capability of that server. }\end{array}$ \\
\hline$l_{l C}$ & $\begin{array}{l}\text { For a given link of } S \times S \text {, this function returns the } \\
\text { capability of that link. }\end{array}$ \\
\hline$l_{l L}$ & $\begin{array}{l}\text { For a given link of } S \times S \text {, this function returns the } \\
\text { latency introduced by that link. }\end{array}$ \\
\hline$G_{I}$ & $\begin{array}{l}\text { The infrastructure modeling graph: } G_{I}=\left(S, L, l_{s C} \text {, }\right. \\
\left.l_{l C}, l_{l L}\right) \text {. }\end{array}$ \\
\hline \multicolumn{2}{|r|}{ Variables and their domains } \\
\hline$V_{M}$ & $\begin{array}{l}\text { The set of the functions of the monitoring service. Its } \\
\text { domain is } D_{V_{M}} \subseteq F \text {. }\end{array}$ \\
\hline$A_{M}$ & $\begin{array}{l}\text { The set of the flows of the monitoring service. Its } \\
\text { domain is } D_{A_{M}} \subseteq F \times F \text {. }\end{array}$ \\
\hline$x_{s f_{i}}$ & $\begin{array}{l}\text { The server which is hosting } f_{i} \in F \text {. The set which } \\
\text { gathers all these variables is } X_{S F}=\left\{x_{s f_{i}} \mid i \in\right. \\
\llbracket 1 ;|F| \rrbracket\} \text {. Their domain is } D_{X_{S F}}=S \text {. }\end{array}$ \\
\hline$x_{l o_{i}}$ & $\begin{array}{l}\text { The set of links which are hosting } o_{i} \in F \times F \text {. The set } \\
\text { which gathers all these variables is } X_{L O}=\left\{x_{l o_{i}} \mid i \in\right. \\
\llbracket 1 ;|F \times F| \rrbracket\} \text {. Their domain is } D_{X_{L O}}=\text { paths }\left(G_{I}\right) .\end{array}$ \\
\hline$x_{o r_{i}}$ & $\begin{array}{l}\text { The flow of the monitoring service which is equivalent } \\
\text { to the flow } r_{i} \in R \text {. The set which gathers all these } \\
\text { variables is } X_{O R}=\left\{x_{o r_{i}}|i \in \llbracket 1 ;| R \mid \rrbracket\right\} \text {. Their } \\
\text { domain is } D_{X_{O R}} \subseteq F \times F \text {. }\end{array}$ \\
\hline \multicolumn{2}{|r|}{ Constraints } \\
\hline Functional & Equations: 1 2] and 3 \\
\hline QoS & 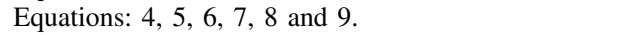 \\
\hline Channeling & Equations: To and 10 \\
\hline \multicolumn{2}{|r|}{ Objective function } \\
\hline$X_{f p}$ & The overall monitoring service footprint (Equation 12) \\
\hline
\end{tabular}

\subsection{Notions and Notations}

We define four notions:

- Function: A function may be either a probe function, a processing function (e.g., filter, aggregate, join) or a user function (e.g., user dashboard, user database). 
- Flow: A flow is the set of data sent from a function to another (along a chain of links that connects them).

- Server: A server is a compute resource. It may be a high performance server in a data center or a user device (e.g., smartphone, home gateway) at the Edge of the infrastructure.

- Link: A link is a network resource that connects the servers.

We represent a directed and labeled graph $G$ as a tuple $G=\left(V, A, l_{1}, l_{2}, \ldots, l_{n}\right)$ such as $V$ is the set of its vertices, $A$ is the set of its $\operatorname{arcs}(A \subseteq V \times V)$ and $l_{1}, l_{2}, \ldots, l_{n}$ are its labeling functions. A labeling function $l_{i}$ may be a vertex labeling function $l_{i}: V \rightarrow L_{i}$ or an arc labeling function $l_{i}: A \rightarrow L_{i}$ such as $L_{i}$ is the set of vertex or arc labels, respectively. In addition, we define the following operators:

- paths(graph): For a given graph, this operator returns all the arcs which form paths.

- source(arcs) and dest(arcs): For a given set of arcs which form a path, these operators return respectively the source vertex and the destination vertex of the path.

- head(arc) and tail(arc): For a given arc, these operators return respectively the head vertex and the tail vertex.

\subsection{Problem Inputs}

The inputs of the problem are the users requirements and the infrastructure description. We model each one of them by a directed graph.

First, we model the users requirements by the directed graph $G_{U}=\left(F, R, l_{f C}, l_{f f}, l_{f S}, l_{f L}, l_{o C}\right)$. Figure 1 depicts an example of this graph. The set of vertices $F$ of $G_{U}$ represents the probes, processing and user functions: $F=P \cup M \cup U$. The set of its arcs $R$ represents the required flows between the different functions. The roles of its labeling functions are detailed in Table 1

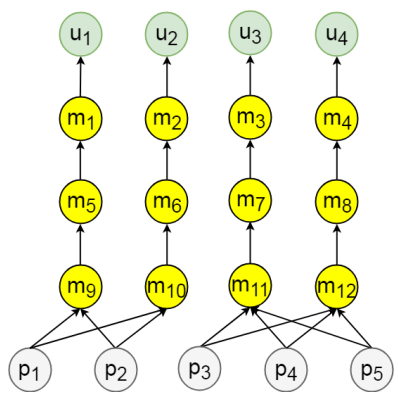

Figure 1. An example of $G_{U}$

Secondly, we model the infrastructure by the directed graph $G_{I}=\left(S, L, l_{s C}, l_{l C}, l_{l L}\right)$. Figure 2 depicts an example of this graph. The set of vertices $S$ of $G_{I}$ represents the servers of the infrastructure. The set of its arcs $L$ represents the network links connecting the servers. Bidirectional links are modeled with two opposed arcs. An arc connects each vertex to itself. It represents a logical link that is intended to host flows between the functions deployed on the same server. The roles of $G_{I}$ labeling functions are detailed in Table 1

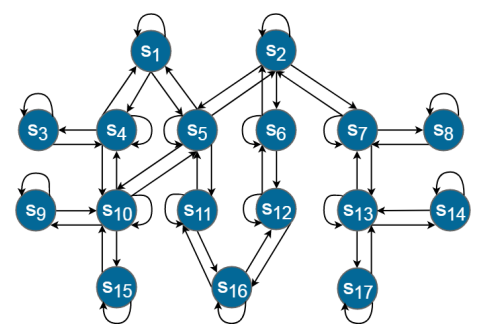

Figure 2. An example of $G_{I}$

\subsection{Variables Definition}

The mutualized placement problem issues four outputs, namely the functions instances, the flows between them, the servers hosting the functions instances and the links hosting the flows. Each element is modeled by a variable. First, we define the variables $V_{M}$ and $A_{M}$. They represent respectively the functions of the monitoring service and the flows between them. Thus, $G_{M}=\left(V_{M}, A_{M}\right)$ is the graph that models the mutualized monitoring service to be determined. Secondly, we define $X_{S F}=\left\{x_{s f_{i}}|i \in \llbracket 1| F \mid, \rrbracket\right\}$ the set of variables $x_{s f_{i}}$ that represent the hosting server of a function $f_{i}, \forall i \in \llbracket 1,|F| \rrbracket$. Finally, we define $X_{L O}=$ $\left\{x_{l o_{i}}|i \in \llbracket 1| F \times F \mid, \rrbracket\right\}$ the set of variables $x_{l_{o_{i}}}$ that represent the set of hosting links of a flow $o_{i}, \forall i \in \llbracket 1,|F \times F| \rrbracket$.

As depicted in Figure 3. $X_{S F}$ and $X_{L O}$ simplify the expression of the $G_{M}$ and $G_{I}$ matching constraints. In order to simplify the expression of the $G_{U}$ and $G_{M}$ matching constraints, we introduce $X_{O R}=\left\{x_{o r_{i}}|i \in \llbracket 1| R \mid, \rrbracket\right\}$ the set of variables $x_{o r_{i}}$ that represent the flows of $G_{M}$ that are functionally equivalent to the flows $r_{i}$ of $G_{U}, \forall i \in \llbracket 1,|R| \rrbracket$.

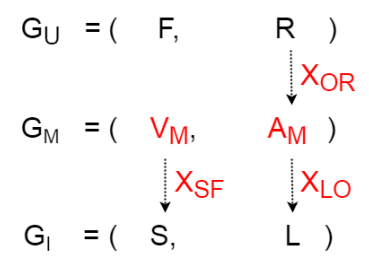

Figure 3. Roles of the variables in graphs matching

Thus, each solution of our problem is an instantiation of $V_{M}, A_{M}, X_{S F}, X_{L O}$ and $X_{O R}$ that minimizes the overall footprint while satisfying simultaneously both the functional and the QoS requirements.

\subsection{Domains Definition}

$G_{M}$ is built from $G_{U}$ because the monitoring service is instantiated according to users requirements. Figure 4 shows an example of a $G_{M}$ that is built from the $G_{U}$ depicted by Figure 1 The differences between them are highlighted in red: a dashed red line is used to mark the vertices and 
the arcs of $G_{U}$ that are deleted in $G_{M}$ and a continuous red line is used to mark the arcs that are added on $G_{M}$. These differences are due to the mutualization of equivalent functions among different users. In fact, functions $m_{9}$ and $m_{10}$ are mutualized among $u_{1}$ and $u_{2}$. In addition, $m_{11}$ and $m_{12}$ are mutualized among $u_{3}$ and $u_{4}$. Finally, $m_{7}$ and $m_{8}$ are mutualized among $u_{3}$ and $u_{4}$.

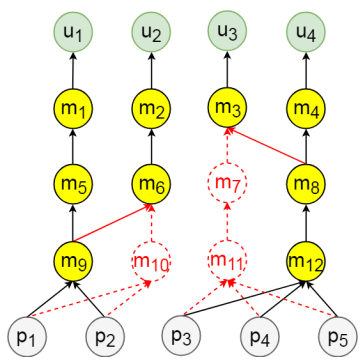

Figure 4. A derivative $G_{M}$ of $G_{U}$

As illustrated in Figure 4, a mutualization of a function $f_{i}$ with a function $f_{j}$ can be described using graph terminology as an edge contraction [11] that consists of two operations. First, each outgoing arc from $f_{j}$ is replaced by an outgoing arc from $f_{i}$ such as the head vertex of the added arc is the same as the head vertex of the arc it is replacing. Similarly, each incoming arc to $f_{j}$ is replaced by an incoming arc to $f_{i}$ such as the tail vertex of the added arc is the same as the tail vertex of the arc it is replacing. Secondly, for each vertex $f_{j}$, the incoming arcs and the outgoing arcs are suppressed.

Hence, the mutualization does not add vertices to $G_{M}$ that are not in $G_{U}$. So, $V_{M} \subseteq F$. Let $D_{V_{M}}$ denote the domain of $V_{M}, D_{V_{M}} \subseteq F$. However, the mutualization may add arcs to $G_{M}$ that are not in $G_{U}$. Thus, $A_{M} \nsubseteq R$. Since $A_{M} \subseteq V_{M} \times V_{M}$ and $V_{M} \subseteq F$ then $A_{M} \subseteq F \times F$. Let $D_{A_{M}}$ denote the domain of $A_{M}, D_{A_{M}} \subseteq F \times F$.

Let $D_{X_{S F}}$ denote the domain of $X_{S F}, D_{X_{S F}}$ is the set of servers that may host the monitoring functions and $D_{X_{S F}}=S$. Let $D_{X_{L O}}$ denote the domain of $X_{L O}, D_{X_{L O}}$ is the set of links that may host the flows between the monitoring functions. To host a flow, the links should form a path. Thus, $D_{X_{L O}}=$ paths $\left(G_{I}\right)$. Finally, let $D_{X_{O R}}$ denote the domain of $X_{O R}, D_{X_{O R}}$ is the set of flows of $G_{M}$ which are equivalent to the flows of $G_{U}$. This set is represented by the variable $A_{M}$. Thus, $D_{X_{O R}}=D_{A_{M}} \subseteq F \times F$.

\subsection{Problem Constraints}

The first three constraints verify the satisfaction of the user functional requirements. They establish the matching between $G_{U}$ and $G_{M}$. First, each users requirements flow $r$ that is incoming to a user $(\operatorname{head}(r) \in U)$ should have an equivalent monitoring service flow $x_{o r}$ that is incoming to that user.

$$
\forall r \in R, \operatorname{head}(r) \notin U \vee \operatorname{head}\left(x_{\text {or }}\right)=\operatorname{head}(r)
$$

Secondly, for each users requirements flow $r$, an equivalent monitoring service flow $x_{\text {or }}$ should be incoming from an identical function to that of $r$.

$$
\forall r \in R, l_{f f}\left(\operatorname{tail}(r), \operatorname{tail}\left(x_{o r}\right)\right)=\operatorname{true}
$$

Finally, for each users requirements flow $r$, an equivalent monitoring service flow $x_{o r}$ should result from the entire required chain of processing functions. Thus, recursively, the incoming flows $x_{o r^{\prime}}$ to $x_{o r}$ should be equivalent to the flows $r^{\prime}$ that are incoming to $r$.

$$
\begin{aligned}
& \forall r \in R, \\
& \forall r^{\prime} \in R, \operatorname{head}\left(r^{\prime}\right) \neq \operatorname{tail}(r) \vee \operatorname{head}\left(x_{o r^{\prime}}\right)=\operatorname{tail}\left(x_{o r}\right)
\end{aligned}
$$

The QoS constraints to satisfy by the monitoring service establish the matching between $G_{M}$ and $G_{I}$. They can be divided into two kinds of constraints. The first kind verifies that the physical architecture of the monitoring service is suitable with the topology of the infrastructure. It is composed of three constraints. First, each flow $o$ of the monitoring service should have hosting links $x_{l o}$.

$$
\forall o \in A_{M}, x_{l o} \neq \varnothing
$$

Secondly, each function of the monitoring service $f \in V_{M}$ that has a predefined hosting server should be hosted on that server (users and probe functions are those which are the most concerned with this constraint because they may have to be hosted near the users or the observed resources, respectively).

$$
\forall f \in V_{M}, l_{f S}(f)=n u l l \vee x_{s f}=l_{f S}(f)
$$

Finally, for each set of links $x_{l o}$ that hosts a flow $o$, the source (the destination, resp.) server of the path they form should host the source (the destination, resp.) function of $o$.

$$
\begin{aligned}
& \forall x_{s f} \in X_{S F}, \\
& \left(\forall o_{1} \in F \times F, \operatorname{head}\left(o_{1}\right) \neq f \vee x_{s f}=\operatorname{dest}\left(x_{l_{o_{1}}}\right)\right) \wedge \\
& \left(\forall o_{2} \in F \times F, \operatorname{tail}\left(o_{2}\right) \neq f \vee x_{s f}=\operatorname{source}\left(x_{l_{o_{2}}}\right)\right)
\end{aligned}
$$

The second kind of QoS constraints are the performance ones. In our case, there are three of them. First, the capability of each hosting server should be satisfied.

$$
\forall s \in S, \sum_{\substack{f \in V_{M} \\ x_{s f}=s}} l_{f C}(f) \leq l_{s C}(s)
$$

Similarly, the capability of each hosting link should be satisfied.

$$
\left.\forall l \in L, \sum_{\substack{\forall o \in A_{M} \\ l \in x_{l o}}} \max _{\substack{\forall r \in R \\ o \in x_{o r}}} l_{o C}(r)\right) \leq l_{l C}(l)
$$

Finally, the latency that is required by the users should be satisfied, along all the paths.

$$
\begin{aligned}
& \forall p \in \operatorname{paths}\left(G_{U}\right), \\
& \forall r \in p, \operatorname{dest}(p) \notin U \vee \sum_{l \in x_{l x_{o r}}} l_{l L}(l) \leq l_{f L}(\operatorname{dest}(p))
\end{aligned}
$$


In addition to the functional and the QoS constraints, we define two channeling constraints. They verify existing dependencies between the variables that do not appear in the previously exposed constraints. First, the set of vertices $V_{M}$ is the set of the heads and tails of the set of arcs $A_{M}$.

$$
V_{M}=\bigcup_{o \in A_{M}}\{\operatorname{head}(o), \operatorname{tail}(o)\}
$$

Secondly, $X_{O R}$ and $A_{M}$ should be equal.

$$
X_{O R}=A_{M}
$$

\subsection{Problem Objective Function}

Mutualizing the processing among different users reduces the compute footprint. However, it may increase the network footprint. In fact, the mutualized processing may be located far from part of users in order to satisfy the latency constraint of others. For this reason, as an objective function, we consider minimizing the monitoring service overall compute and network footprint rather than maximizing the mutualization. We evaluate the sum of both footprints by the variable $X_{f p}$ to minimize. We consider that both footprints have the same cost, but depending on the context, different coefficients may be used in this sum.

$$
\left.X_{f p}=\sum_{\substack{s \in S \\ f \in V_{M} \\ x_{s f}=s}} l_{s C}(f)+\sum_{\substack{l \in L \\ o \in A_{M} \\ l \in x_{l_{o}}}} \max _{\substack{\forall r \in R \\ o \in x_{o r}}} l_{o C}(r)\right)
$$

\section{Evaluation}

In order to evaluate the relevance of our approach, we compare the mutualized placement $(M)$ with the nonmutualized placement $(N M)$ regarding their footprint on the infrastructure and their required calculation time.

To calculate the $N M$, we modified the model by removing the matching between $G_{U}$ and $G_{M}$ and considering that $G_{M}=G_{U}$. Thus, the variables $V_{M}, A_{M}$ and $x_{o r_{i}} \mid$ $i \in \llbracket 1 ;|R| \rrbracket$ are replaced by $F, R$ and $r_{i}|i \in \llbracket 1 ;| R \mid \rrbracket$, respectively. In addition, Constraints 1, 2, 3, 10 and 11 related to these variables, are removed. We keep the same objective function. Thus, the resources usage is still minimized but without functions and flows mutualization.

In the calculation of both placements, a strategy is defined to explore the search space. It is a combination of the "last conflict" [15] and the "smallest domain first" strategies. It prioritizes the selection of the variables that were involved in the last conflict. If there are no conflicts, the strategy selects the variables having the smallest domains and assigns to them the lowest values in their domains. We implemented both placement models using the Choco [16] 4.0.6 constraint solver. The source code is available online : https: //github.com/edgeMonitoring/PlacementCalculator. We performed all the experiments on a machine with a Xeon E5$2640 \mathrm{~V} 4$ processor and $64 \mathrm{~GB}$ of RAM using the Ubuntu 16.04 operating system and a Java 1.8.0 virtual machine.

\subsection{Test Scenarios}

We consider two test categories to analyze separately the impact of users requirements changes (Test UR1 and Test UR2) and the impact of infrastructure changes (Test I1 and Test I2).

Regarding users requirements, Tests UR1 and UR2 consider a basic users requirements $G_{U}$, where all users need to perform the same number of processing functions on a common probe. In Test UR1, we vary the number of the required processing functions per user $(|\mathrm{M}| / \mid \mathrm{UI})$ while keeping the number of users fixed to $4(|\mathrm{U}|=4)$ as depicted in Figure 5 (a). Conversely, in Test UR2, we vary the number of users (IUI) while keeping the number of the required processing functions per user fixed to $3(|\mathrm{M}| /|\mathrm{U}|=3)$ as depicted in Figure 5 (b). We assume that each function requires $1 \mathrm{MB}$ of RAM and that each communication between the functions requires $1 \mathrm{Mbps}$ of bandwidth (these values have been arbitrary chosen). We generate the functions types and parameters randomly with three assumptions. First, the predefined hosting server of a probe function is an edge device (when such a device exists in the infrastructure). Secondly, between the predefined hosting servers of each user and its probes, there is at least one path whose latency is inferior or equal to the maximum tolerated by that user. Finally, $60 \%$ of the processing functions that have the same rank in the different processing chains are identical (the outgoing flows of these functions satisfy Constraint 21. As a matter of fact, this assumption does not determine the expected mutualization rate because it does not guarantee the satisfaction of the rest of the functional constraints (i.e., Constraints 1 and 3). For both tests, the infrastructure is composed of 4 trees of servers and it has the same configuration with Test I1 infrastructures that is detailed in the following paragraph.

Regarding the impact of the infrastructure, Test I1 and Test I2 are based on two different infrastructure topologies. In Test I1, the topology is a ring of trees. Each tree is composed of 7 servers: a central point of presence $\left(c_{i}\right)$, two regional points of presence $\left(r_{i}\right)$ and four edge devices $\left(e_{i}\right)$ such as each $c_{i}$ is connected to two $r_{i}$ and each $r_{i}$ is connected to two $e_{i}$ (We model each point of presence by a high-performance server because the latency inside a data center is lower than $1 \mathrm{~ms}$ [17]). In this test, we vary the number of trees (ISI/7) as depicted in Figure 5 (c). In Test I2, we consider a partially connected topology. We take as example a part of the topology of RENATER, the French NREN [18]. It is composed of eight central points of presence $c_{1} . . c_{8}$, which are located in Rennes, Paris, Strasbourg, Bordeaux, Lyon, Toulouse, Marseille and Nice, respectively as well as a regional point of presence $r$ located in Orsay. Figure 5 (d) depicts this infrastructure. For Test I1, we assume that the latencies of the links interconnecting the different $c_{i}$, connecting a $c_{i}$ with an $r_{i}$ and connecting an $r_{i}$ with an $e_{i}$ are $22 \mathrm{~ms}, 12 \mathrm{~ms}$ and $8 \mathrm{~ms}$, respectively. For Test I2, we consider the links latencies that are afforded by RENATER [19]. For both kinds of infrastructures, we assume that the available hosting capabilities of the $c_{i}, r_{i}$ and 


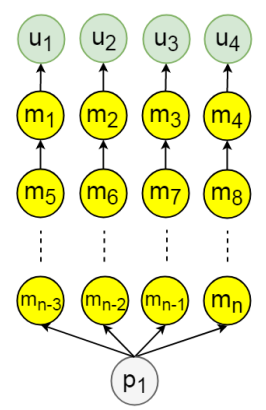

(a) Test UR1

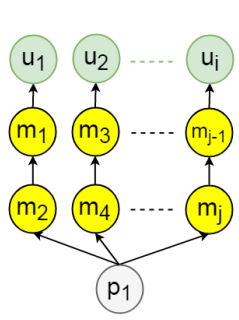

(b) Test UR2

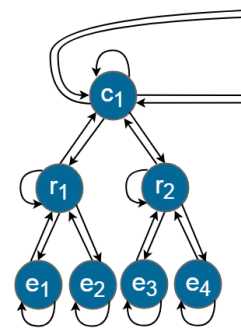

Figure 5. Tests inputs

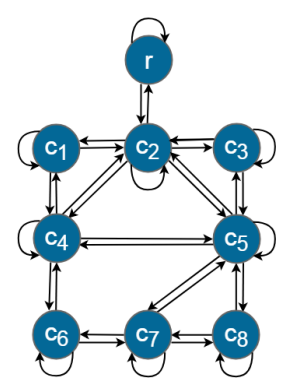

(d) Test 12

(c) Test I1

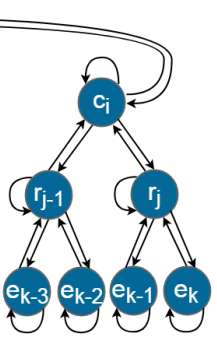
(1)

$e_{i}$ are $10 \mathrm{~GB}, 1 \mathrm{~GB}$ and $100 \mathrm{MB}$, respectively. In addition, we consider that the bandwidths of the links interconnecting the $c_{i}$, connecting a $c_{i}$ with an $r_{i}$ and connecting an $r_{i}$ with an $e_{i}$ are $10 \mathrm{Gbps}, 1 \mathrm{Gbps}$ and $100 \mathrm{Mbps}$, respectively. These values of the hosting capabilities have been chosen to guarantee the existence of a $N M$. Otherwise, it may be possible to find $M$ solutions and no $N M$ ones. For the sake of simplicity, we do not consider this case. In both Tests I1 and $\mathrm{I} 2$, we assume that there is no routing restrictions (This is the most challenging configuration since it maximizes the number of network paths). Finally, the users requirements input is composed of 4 users ( $|\mathrm{U}|=4)$ each requiring to perform 3 processing functions $(|\mathrm{M}| /|\mathrm{U}|=3)$ on the same probe. The compute and network capabilities it requires are as those of Tests UR1 and UR2 users requirements.

\subsection{Test Results}

In this section, we compare the $M$ and the $N M$ approaches based on the calculation time that they require as well as their compute and their network footprint. We consider the first and last solutions found within 10 minutes. Results are depicted in Table 2 and Table 3. Each value in these tables is the mean of the results of 30 experiments that differ in the randomly generated parameters. The footprint gain realized by the $M$ is represented as a percentage of the $N M$ footprint. In $3 \%$ of the experiments of Test I1 where the number of trees $(|\mathrm{S}| / 7)$ is equal to 2 , the search space could be entirely explored during the calculation of the $N M$. Meanwhile, 10 minutes were not enough to find an $M$ solution in $13 \%$ of the experiments of Test UR1 where $|\mathrm{M}| /|\mathrm{U}|=6$ and in $27 \%$ of the experiments of Test UR1 where $|\mathrm{M}| /|\mathrm{U}|=8$.

3.2.1. Footprint Analyses. The $N M$ results show that the compute footprint is the same in the first and the last solutions. It is equal to the sum of the compute capabilities that are required by the functions (i.e., $|F| \times 1 M B$ ) because there is no functions mutualization. For the $M$ results, the compute footprint is also the same in the first and the last solutions but it is lower than the sum of the compute capabilities that are required by the functions. Thus, a $G_{M}$ is found. However, it is the only one because the calculation of its possible placements requires more than 10 minutes as in the most cases for $N M$.

Since for both placements, the first and the last solutions have the same number of functions, these solutions have also the same number of flows. However, for both placements, the last solution has a lower network footprint than the first one. This is due to the fact that the solver manages to place the flows on a lower number of links. Test I1 shows how the number of links connecting the servers affects the network footprint since for the same users requirements input, the network footprint increases when the scale of the infrastructure increases.

As expected, the first and the last solutions of the $M$ have less compute and network footprint than the first and the last solutions of the $N M$, respectively. The realized gain for the compute footprint ranges from $-8 \%$ to $-28 \%$ and it ranges from $-10 \%$ to $-27 \%$ for the network one. In most cases, the first solution of the $M$ has even less compute and network footprints than the last solution of the $N M$. This is due to the fact that the $M$ solutions are not attainable by the $N M$ calculation.

3.2.2. Calculation Time Analyses. The inputs scale affects significantly the calculation time. Table 2 shows that the time required to find the first solutions increases exponentially when the input scale increases linearly. This is due to the NP-completeness of the $M$ and $N M$ problems.

For the different tests, Table 2 shows that the first solution of the $M$ case required more time than the one of the $N M$ case. In addition, as detailed previously, 10 minutes were not enough to find a solution in some experiments for the $M$ calculation. Meanwhile, they were enough in other experiments to explore the search space entirely for the $N M$ calculation. This is due to the fact that the $M$ problem complexity is higher than the $N M$ one. The former consists of two NP-complete problems (i.e., edge contraction and graph matching) whereas the latter consists of only one (i.e., graph matching). Since any $N M$ is a viable solution for the $M$ calculation, it is possible to begin the $M$ calculation by looking for an $N M$. This comes down to adding this constraint: $\forall r \in R, \operatorname{head}\left(x_{o r}\right)=\operatorname{head}(r) \wedge \operatorname{tail}\left(x_{o r}\right)=\operatorname{tail}(r)$ and to relaxing it once a solution is found. Thus, it is possible to reduce the $M$ calculation time. 


\begin{tabular}{|c|c|c|c|c|c|c|c|c|c|c|}
\hline \multirow{2}{*}{ Test } & \multicolumn{4}{|c|}{ Inputs } & \multicolumn{2}{|c|}{ Time(s) } & \multicolumn{2}{|c|}{ Compute fp.(MB) } & \multicolumn{2}{|c|}{ Network fp.(Mbps) } \\
\hline & $|\mathbf{M}| /|\mathbf{U}|$ & $|\mathbf{U}|$ & $|\mathbf{F}|$ & $|\mathbf{S}| / 7$ & NM & M & NM & M & NM & M \\
\hline \multirow{4}{*}{ UR1 } & 2 & 4 & 13 & 4 & 0.034 & 1.878 & 13 & $10(-23 \%)$ & 24 & $19(-21 \%)$ \\
\hline & 4 & 4 & 21 & 4 & 0.085 & 27.935 & 21 & $17(-19 \%)$ & 32 & $26(-19 \%)$ \\
\hline & 6 & 4 & 29 & 4 & 0.153 & 56.985 & 29 & $25(-14 \%)$ & 40 & $34(-15 \%)$ \\
\hline & 8 & 4 & 37 & 4 & 0.187 & 63.16 & 37 & $32(-14 \%)$ & 48 & $42(-13 \%)$ \\
\hline \multirow{4}{*}{ UR2 } & 3 & 3 & $\overline{13}$ & $\overline{4}$ & 0.046 & 1.542 & 13 & $12(-8 \%)$ & 21 & $19(-10 \%)$ \\
\hline & 3 & 5 & 21 & 4 & 0.078 & 3.988 & 21 & $18(-14 \%)$ & 35 & $30(-14 \%)$ \\
\hline & 3 & 7 & 29 & 4 & 0.106 & 16.527 & 29 & $21(-28 \%)$ & 50 & $38(-24 \%)$ \\
\hline & 3 & 9 & 37 & 4 & 0.148 & 32.98 & 37 & $28(-24 \%)$ & 64 & $50(-22 \%)$ \\
\hline \multirow{4}{*}{ I1 } & 3 & 4 & $\overline{17}$ & 2 & 0.007 & 0.276 & 17 & $13(-24 \%)$ & 26 & $19(-27 \%)$ \\
\hline & 3 & 4 & 17 & 4 & 0.078 & 6.578 & 17 & $13(-24 \%)$ & 28 & $22(-21 \%)$ \\
\hline & 3 & 4 & 17 & 6 & 0.191 & 29.475 & 17 & $13(-24 \%)$ & 31 & $25(-19 \%)$ \\
\hline & 3 & 4 & 17 & 8 & 0.405 & 99.301 & 17 & $13(-24 \%)$ & 33 & $26(-21 \%)$ \\
\hline $\mathrm{I} 2$ & 3 & 4 & 17 & $9 / 7$ & 0.008 & 0.358 & 17 & $13(-24 \%)$ & 19 & $15(-21 \%)$ \\
\hline
\end{tabular}

\begin{tabular}{|c|c|c|c|c|c|c|c|c|c|c|}
\hline \multirow{2}{*}{ Test } & \multicolumn{4}{|c|}{ Inputs } & \multicolumn{2}{|c|}{ Time(s) } & \multicolumn{2}{|c|}{ Compute fp.(MB) } & \multicolumn{2}{|c|}{ Network fp.(Mbps) } \\
\hline & $|\mathbf{M}| / \mathbf{U} \mid$ & |U| & $|\mathbf{F}|$ & $|\mathbf{S}| / 7$ & NM & M & NM & M & NM & $\mathrm{M}$ \\
\hline \multirow{4}{*}{ UR1 } & 2 & 4 & 13 & 4 & 223.298 & 59.095 & 13 & $10(-23 \%)$ & 20 & $16(-20 \%)$ \\
\hline & 4 & 4 & 21 & 4 & 186.944 & 157.614 & 21 & $17(-19 \%)$ & 29 & $24(-17 \%)$ \\
\hline & 6 & 4 & 29 & 4 & 119.902 & 166.238 & 29 & $25(-14 \%)$ & 38 & $32(-16 \%)$ \\
\hline & 8 & 4 & 37 & 4 & 88.282 & 102.676 & 37 & $32(-14 \%)$ & 46 & $41(-11 \%)$ \\
\hline \multirow{4}{*}{ UR2 } & 3 & 3 & 13 & 4 & 232.378 & 252.853 & 13 & $12(-8 \%)$ & 17 & $15(-12 \%)$ \\
\hline & 3 & 5 & 21 & 4 & 218.736 & 173.373 & 21 & $18(-14 \%)$ & 31 & $27(-13 \%)$ \\
\hline & 3 & 7 & 29 & 4 & 176.994 & 192.797 & 29 & $21(-28 \%)$ & 46 & $35(-24 \%)$ \\
\hline & 3 & 9 & 37 & 4 & 116.676 & 93.053 & 37 & $28(-24 \%)$ & 60 & $49(-18 \%)$ \\
\hline \multirow{4}{*}{ I1 } & 3 & $\overline{4}$ & 17 & $\overline{2}$ & 277.179 & 63.126 & 17 & $13(-24 \%)$ & 19 & $15(-21 \%)$ \\
\hline & 3 & 4 & 17 & 4 & 231.245 & 153.024 & 17 & $13(-24 \%)$ & 24 & $19(-21 \%)$ \\
\hline & 3 & 4 & 17 & 6 & 120.906 & 221.019 & 17 & $13(-24 \%)$ & 28 & $22(-21 \%)$ \\
\hline & 3 & 4 & 17 & 8 & 82.793 & 227.968 & 17 & $13(-24 \%)$ & 30 & $24(-20 \%)$ \\
\hline $\mathrm{I} 2$ & 3 & 4 & 17 & $9 / 7$ & 95.717 & 20.37 & 17 & $13(-24 \%)$ & 17 & $13(-24 \%)$ \\
\hline
\end{tabular}

For the same number of functions, Table 2 shows that it is harder for both placements to find a first solution in the tests having the higher number of processing functions. In fact, these functions introduce more unknowns to the problem than the other functions (i.e., users and probes) since it is unknown whether they have to be mutualized or not. In addition, their hosting servers are unknown.

Compared to an infrastructure of Test I1 that is composed of two trees $(|S|=14,|\mathrm{~L}|=40)$, the infrastructure of Test I2 has a lower number of servers and links $(|\mathrm{S}|=9$, $|\mathrm{L}|=35)$. However, Table 2 shows that for both placements, it is harder to find the first solution in the latter test than in the former. This is due to the difference of the infrastructures topologies, which results in different numbers of network paths. In fact, for the infrastructure of Test I1 that is composed of 2 trees, there is only one path between any pair of servers. However, for the infrastructure of Test I2, there are at least two paths between any pair of servers except $c_{2}$ and $r$, which are connected with one path only.

\section{Related Work}

Sharing sensors information among different tenants has been identified in the IoT literature as an opportunity to increase the business value [20]. In order to promote it, different studies have been conducted. For instance, a threelayered architecture has been proposed to abstract the IoT devices heterogeneity [21], a metadata model has been proposed to unify the IoT data description [22] and semantic methods have been proposed to identify similarity between data sent by heterogeneous sensors [23]. These studies deal only with sharing the sensors raw measurements. However, they do not address sharing the measurements processing.

Processing unbounded streams of data while considering the Edge infrastructure capabilities has been recently studied in the stream processing literature. The latter is a very active research in data mining. In [24], relying on Edge infrastructures to process data streams near to the users is investigated. In [25], the authors formalize the problem of placing stream processors in an Edge infrastructure to meet users requirements while considering the infrastructure capabilities. However, these works do not consider the possibility of mutualising processing among different tenants.

The mutualisation concept has been proposed in the network service function chaining problem [26]. It consists in efficiently passing users network flows through a sequence of network functions (e.g., firewall, intrusion detection systems, load balancer...) to reduce the overall network service footprint. In this context, studies as [27] and [28] propose models where the same network function is dedicated to process different users flows. Thus, the allocated hosting resources are used more efficiently since their idle time is reduced. However, these works do not specifically consider the case of users having identical flows. Consequently, such flows are processed redundantly.

To the best of our knowledge, we are the first to propose mutualising identical processing and flows among different tenants to reduce the Edge infrastructure footprint. 


\section{Conclusion and Future Work}

In this paper, we investigated the footprint challenge of Edge infrastructures monitoring. To address this issue, we proposed to mutualize identical processing and flows among different tenants. Our approach is motivated by the multitenant aspect of the Edge infrastructure since the tenants sharing the same resources may have the same monitoring interests.

We formalized the mutualization determination as a constraint satisfaction problem whose objective is to reduce the overall monitoring service footprint while meeting the QoS requirements of each tenant.

The micro-benchmarks we performed showed the pertinence of our approach in reducing the monitoring footprint. However, because of the NP-completeness of the problem, the mutualization increases the placement calculation time. To cope with this limit, it is possible to target a nonmutualised placement as a first solution then continue the search for a mutualised one. In addition, in order to significantly reduce the calculation time, we envision to decentralize the mutualization calculation. To assign the inputs to the different calculators, we intend to consider the inputs nature in addition to their scale as our evaluation showed that the rate of the processing functions in the users requirements and the topology of the infrastructure affect the duration of the calculation. Finally, to cope with the dynamicity of the infrastructure (mobile Edge devices, virtual machines with short life cycles), we envision to rely on dynamic resolution methods [29].

\section{References}

[1] L. Atzori, A. Iera, and G. Morabito, "The internet of things: A survey," Computer networks, vol. 54, no. 15, pp. 2787-2805, 2010.

[2] A. Ahmed and E. Ahmed, "A survey on mobile edge computing," in 2016 10th International Conference on Intelligent Systems and Control (ISCO), Jan 2016, pp. 1-8.

[3] D. Kreutz, F. Ramos, P. Esteves Verissimo, C. Esteve Rothenberg, S. Azodolmolky, and S. Uhlig, "Software-defined networking: A comprehensive survey," Proceedings of the IEEE, vol. 103, no. 1, pp. 14-76, Jan. 2015.

[4] R. Mijumbi, J. Serrat, J.-L. Gorricho, N. Bouten, F. De Turck, and R. Boutaba, "Network function virtualization: State-of-the-art and research challenges," IEEE Communications Surveys \& Tutorials, vol. 18, no. 1, pp. 236-262, 2015.

[5] W. Shi, J. Cao, Q. Zhang, Y. Li, and L. Xu, "Edge computing: Vision and challenges," IEEE Internet of Things Journal, vol. 3, no. 5, pp. 637-646, 2016.

[6] A. Lebre, J. Pastor, A. Simonet, and F. Desprez, "Revising openstack to operate fog/edge computing infrastructures," in Cloud Engineering (IC2E), 2017 IEEE International Conference on. IEEE, 2017, pp. $138-148$.

[7] A. Asta, "Observability at twitter: technical overview, part i, 2016," https://blog.twitter.com/engineering/en_us/a/2016/ observability-at-twitter-technical-overview-part-i.html accessed: 2018-10-01.

[8] M. P. Andersen and D. E. Culler, "Btrdb: Optimizing storage system design for timeseries processing." in FAST, 2016, pp. 39-52.
[9] L. Romano, D. De Mari, Z. Jerzak, and C. Fetzer, "A novel approach to qos monitoring in the cloud," in Data Compression, Communications and Processing (CCP), 2011 First International Conference on. IEEE, 2011, pp. 45-51.

[10] M. Abderrahim, M. Ouzzif, K. Guillouard, J. Francois, and A. Lebre, "A holistic monitoring service for fog/edge infrastructures: A foresight study," in 2017 IEEE 5th International Conference on Future Internet of Things and Cloud (FiCloud), Aug 2017, pp. 337-344.

[11] T. Asano and T. Hirata, "Edge-deletion and edge-contraction problems," in Proceedings of the fourteenth annual ACM symposium on Theory of computing. ACM, 1982, pp. 245-254.

[12] E. Bengoetxea, "Inexact graph matching using estimation of distribution algorithms," Ph.D. dissertation, Ecole Nationale Supérieure des Télécommunications, Paris, France, Dec 2002.

[13] A. M. Abdulkader, "Parallel algorithms for labelled graph matching," $\mathrm{Ph} . \mathrm{D}$. dissertation, Colorado School of Mines, 1998

[14] V. Kumar, "Algorithms for constraint-satisfaction problems: A survey," AI magazine, vol. 13, no. 1, p. 32, 1992.

[15] C. Lecoutre, L. Saïs, S. Tabary, and V. Vidal, "Reasoning from last conflict (s) in constraint programming," Artificial Intelligence, vol. 173, no. 18, pp. 1592-1614, 2009.

[16] N. Jussien, G. Rochart, and X. Lorca, "Choco: an open source java constraint programming library," in CPAIOR'08 Workshop on Open-Source Software for Integer and Contraint Programming (OSSICP'08), 2008, pp. 1-10.

[17] S. M. Rumble, D. Ongaro, R. Stutsman, M. Rosenblum, and J. K. Ousterhout, "It's time for low latency." in HotOS, vol. 13, 2011.

[18] V. Schafer, "Part of a whole: Renater, a twenty-year-old network within the internet," Information \& Culture, vol. 50, no. 2, 2015.

[19] "IPv4 MULTICAST Service," https://pasillo.renater.fr/test/get_ qosmetrics_resultsMULTICASTv4.php (Accessed: 2018-10-01).

[20] S. Jernigan, D. Kiron, and S. Ransbotham, "Data sharing and analytics are driving success with iot," MIT Sloan Management Review, vol. 58, no. $1,2016$.

[21] Y. Benazzouz, C. Munilla, O. Gunalp, M. Gallissot, and L. Gurgen, "Sharing user iot devices in the cloud" in Internet of Things (WFIoT), 2014 IEEE World Forum on. IEEE, 2014, pp. 373-374.

[22] B. Xu, L. Da Xu, H. Cai, C. Xie, J. Hu, and F. Bu, "Ubiquitous data accessing method in iot-based information system for emergency medical services," IEEE Transactions on Industrial Informatics, vol. 10, no. 2, pp. 1578-1586, 2014.

[23] M. Antunes, D. Gomes, and R. L. Aguiar, "Towards iot data classification through semantic features," Future Generation Computer Systems, 2017.

[24] M. D. de Assuncao, A. da Silva Veith, and R. Buyya, "Distributed data stream processing and edge computing: A survey on resource elasticity and future directions," Journal of Network and Computer Applications, vol. 103, pp. 1-17, 2018.

[25] V. Cardellini, V. Grassi, F. Lo Presti, and M. Nardelli, "Optimal operator replication and placement for distributed stream processing systems," ACM SIGMETRICS Performance Evaluation Review, vol. 44, no. 4, pp. 11-22, 2017.

[26] J. Halpern and C. Pignataro, "Service function chaining (sfc) architecture," Tech. Rep., 2015.

[27] M. C. Luizelli, L. R. Bays, L. S. Buriol, M. P. Barcellos, and L. P. Gaspary, "Piecing together the nfv provisioning puzzle: Efficient placement and chaining of virtual network functions," in Integrated Network Management (IM), 2015 IFIP/IEEE International Symposium on. IEEE, 2015, pp. 98-106.

[28] N. Tastevin, M. Obadia, and M. Bouet, "A graph approach to placement of service functions chains," in Integrated Network and Service Management (IM), 2017 IFIP/IEEE Symposium on. IEEE, 2017.

[29] R. J. Wallace, D. Grimes, and E. C. Freuder, "Solving dynamic constraint satisfaction problems by identifying stable features." in IJCAI, vol. 9, 2009, pp. 621-627. 